\section{LUCADA: a valuable resource but there are questions it cannot answer}

\author{
Tom Treasure, ${ }^{1}$ Gunnar Hillerdal, ${ }^{2}$ Martin Utley ${ }^{1}$
}

Rich and colleagues have recently provided information on lung cancer in England from the National Lung Cancer Audit database (LUCADA). ${ }^{1}$ The authors concluded that LUCADA accurately describes people in England with lung cancer and can be used to drive healthcare improvements. They found that the socioeconomic status of individual patients does not affect survival and has only limited impact on patients' access to treatment. The question remained as to whether differences in service, at the level of the NHS Trust, might explain regional variation in outcome. In this issue of Thorax, they explore inequalities in outcomes and how these are influenced by clinical characteristics of the patients and the features of local cancer services. In the tables, they also show that survival (but not the proportion operated on) differs between various ethnic groups but do not comment further on this finding in the results or discussion sections. The authors conclude that there is an opportunity to increase access to thoracic surgeons for patients who present and are being cared for in non-surgical centres. ${ }^{2}$

Access to care should ideally not be dependent on where people live. There is no doubt that historically it has been and the authors quote evidence in support of the point from Scotland, ${ }^{3}$ where population density is low and thoracic units are geographically sparse. In the major cities of England whether patients are seen in a specialist thoracic centre is not determined solely by where they live. Selective routing of patients by general practitioners could contribute to the differences in utilisation of surgery reported. The authors have not presented an analysis by

${ }^{1}$ Clinical Operational Research Unit, Department of Mathematics, University College London, London, UK; ${ }^{2}$ Department of Lung Medicine and Allergy, Karolinska Hospital, Stockholm, Sweden

Correspondence to Professor Tom Treasure, Department of Mathematics, University College London, Clinical Operational Research Unit, 4 Taviton Street, London WC1, London, UK; tom.treasure@gmail.com geography, although they refer to variation in outcome being anecdotally attributed to geographical differences in patient features. The authors' access to the postcode data required to calculate Townsend scores suggests that this analysis could be done. able overlap in the service provision for cardiac and thoracic surgery. Core training is in both cardiac and thoracic elements of the specialty and many surgeons continue in consultant practice doing both. For a period in history, coronary artery surgery consumed the time, energy and resources of cardiothoracic units through the 1980s and 1990s. With striking reductions in coronary artery disease in the British Isles ${ }^{4}$ almost completely attributable to changes in life style, diet and public health measures, the pendulum may be swinging back to mixed cardiothoracic practice. By the far from perfect measure of perioperative mortality, there was no evident difference between the outcomes for solely thoracic and mixed cardiothoracic surgeons ${ }^{5}$ but there can be little doubt that while there was a drive to provide equitable access to coronary interventions, provision of lung cancer surgery took something of a back seat. ${ }^{6} 7$ This problem has been addressed and considerable effort has gone into improving organisation of cancer services. No suggestion is made by Rich et al that there are insufficient pairs of surgical hands to provide an appropriate level of lung cancer surgery in both quantity and quality.

Specialist services such as surgery and radiotherapy are centralised according to the hub and spoke system referred to in the paper. $^{2}$ The analysis seems to show that the system is working for patients and probably strikes an appropriate balance between the conflicting pressures of accessibility of surgery and the need for centralisation of facilities and expertise. There may yet be room for improvement in equity of access to thoracic surgery in the UK, but that this would confer a survival advantage cannot be derived
In British practice, there is a consider- from these data. The analysis shows only a small, non-significant difference in survival ( $82 \%$ vs $79 \%$ alive at the date of last data collection in September 2009) depending on whether or not a patient is first seen in a surgical centre. Patients seen at specialist centres are more likely to have investigations more expeditiously and a definitive tissue diagnosis made earlier. This potential lead-time bias (ie, measured survival being longer simply because of an earlier diagnosis) might be all the difference there is.

The observation that tumour doubling times are widely variable as deduced by Geddes from serial chest radiographs ${ }^{8}$ has been revisited in the modern era by studying patients in a screening programme who had two or more CT scans at intervals. ${ }^{9}$ Some of these patients had doubling times of 1000 or more days. The point to remember in interpreting these data is that it takes 10 doubling times to go from the readily seen $1 \mathrm{~cm}$ nodule to a lethal cancer load; in some patients, given these doubling times, this would take 30 years. ${ }^{10}$ Thus, surgical outcomes for screened patients and chance pick-ups on x-rays, taken for other reasons, are not comparable with cancer survival among cohorts that present with clinical symptoms. With screened detection in asymptomatic patients, the clinical picture and the expectations that are conveyed by the diagnosis of 'lung cancer' are different; the diagnosis is being reframed. ${ }^{11}$ Outcomes in a series of patients identified by screening cannot be directly compared with those in whom investigations have been initiated after clinical presentation. It is in the nature of any viable cancer screening study that it will increase the number of cases detected and the number of cases actively managed. Prior to the NLST study, this had not translated into an improvement in survival in lung cancer. ${ }^{12}$ The NLST study shows a difference of 62 deaths per 100000 person-years with CT screening compared with screening with plain chest radiograph. This was at the cost of a higher false positive rate of $96.4 \%$ in the low-dose CT group compared with $94.5 \%$ in the radiography group.

The assumption implicit in the paper by Rich and colleagues, that operating on a higher proportion of patients diagnosed in the modern era with lung cancer will increase the number of those who benefit merits caution. When surgeons operated on the basis of a chest x-ray and a bronchoscopy, the 5-year survival was $25 \%-$ $27 \%$ based on data from six high volume expert surgeons who operated on 9000 
patients for lung cancer between 1949 and $1980 .^{13}$ The survival rate had remained stable over those 30 years. Fibreoptic bronchoscopy, mediastinoscopy, CT scans and positron emission tomography (PET) have successively excluded patients in whom complete resection of cancer cannot be achieved. This more restrictive selection of patients for operation contributed to the much higher proportion of 5-year survivors (about double) in many subsequent reported surgical followup studies and in contemporary reported survival data. However, in LUCADA data ${ }^{2}$ only the upper quartile of operated patients were alive at 2150 days, which translates to a 6-year survival of under $25 \%$, a very similar overall survival to that attained years ago. ${ }^{13}$ Having narrowed down surgical practice by being selective, are practitioners losing the ground that had been gained by assuming that more operations equates to better care? Are we repeating the experience of surgeons who operated in the era before mediastinoscopy, CT and $\mathrm{PET}^{13}$ by knowingly extending the surgical boundaries?

The authors find that there is a $17 \%$ surgery rate in patients first seen at a surgical centre versus a $12 \%$ rate for those initially referred to non-surgical centres. The implicit assumption that the higher figure is to be preferred deserves critical evaluation. Clinical teams know how to pick winners and the finding that patients in LUCADA who were selected to have surgery lived longer than those who were not selected is maybe evidence of good selection, but cannot be viewed, of itself, as proving the beneficial effect of surgery. It is probable that teams involved in the management of lung cancer can identify, even within any given stage, those patients with a higher likelihood of longer survival. The reported stage, however carefully determined, is fixed in time in a database but the rate at which patients moved into and through that stage is critical, uncaptured information that can be very evident to a clinician assessing a patient over a couple of weeks. Expanding surgery on the basis of the generally valuable but uncontrolled observational data provided by LUCADA may simply lead to more unavailing surgery.

We should remember that after years of ever more radical surgery for breast cancer, doubt about its appropriateness began to grow but it was a randomised controlled trial that allowed clinicians to confidently put that era behind them. ${ }^{14}$ The difference reported by Rich and colleagues between the $12 \%$ surgery rate in patients not initially referred to a surgical centre and the $17 \%$ for those first seen at a surgical centre includes patients with $\mathrm{N} 2$ or other more advanced disease. The apparently never-ending N2 debate (as one example-there are other areas of uncertainty) is surely amenable to carefully considered randomised trials. Such trials would necessarily be of multimodality treatment strategies with or without surgery but this is all the more reason for randomised trials because however sophisticated the analysis of registry data, we cannot be sure of the signal among the noise. ${ }^{15}$ Judging whether it is time for surgical interventions to advance, to retreat or to hold their ground requires better evidence; using LUCADA to plan trials would be preferable to encouraging the creeping extension of major surgery based on observational data.

\section{Competing interests None.}

Contributors All authors were involved in the drafting of the work and agreed on the final version.

Provenance and peer review Commissioned; internally peer reviewed.

Data sharing statement We have provided no original data in our contribution.

Published Online First 13 September 2011

Thorax 2011;66:1023-1024.

doi:10.1136/thoraxjnl-2011-200922

\section{REFERENCES}

1. Rich AL, Tata LJ, Stanley RA, et al. Lung cance in England: information from the National Lung Cancer Audit (LUCADA). Lung Cancer 2011;72:16-22

2. Rich $\mathbf{A L}$, Tata $\mathrm{LJ}$, Free $\mathbf{C M}$, et al. Inequalities in outcomes for non-small cell lung cancer: the influence of clinical characteristics and features of the local lung cancer service. Thorax 2011;66: 1078-84.

3. Campbell NC, Elliott AM, Sharp L, et al. Rural factors and survival from cancer: analysis of Scottish cancer registrations. $\mathrm{Br} J$ Cancer 2000;82:1863-6.

4. Mirzaei M, Truswell AS, Taylor R, et al. Coronary heart disease epidemics: not all the same. Heart 2009;95:740-6

5. Treasure T, Utley M, Bailey A. Assessment of whether in-hospital mortality for lobectomy is a useful standard for the quality of lung cancer surgery: retrospective study. BMJ 2003;327:73.

6. Partridge MR. Thoracic surgery in a crisis. BMJ 2002;324:376-7.

7. Treasure T, Dussek J, Eraut D, et al. The Critical Under-Provision of Thoracic Surgery in the UK: Report of a joint Working Group of The British Thoracic Society and The Society of Cardiothoracic Surgeons of Great Britain and Ireland. Society of Cardiothoracic Surgeons of Great Britain and Ireland. Society of Cardiothoracic Surgeons of Great Britain \& Ireland, 2002. Ref Type: Report. http://www.scts.org/doc/ 6168.

8. Geddes DM. The natural history of lung cancer: a review based on rates of tumour growth. $\mathrm{Br} \mathrm{J}$ Dis Chest 1979;73:1-17.

9. Lindell RM, Hartman TE, Swensen SJ, et al. Fiveyear lung cancer screening experience: CT appearance, growth rate, location, and histologic features of 61 lung cancers. Radiology 2007;242:555-62.

10. Hillerdal G. Indolent lung cancers-time for a paradigm shift: a review. J Thorac Oncol 2008;3:208-11.

11. Rosenberg CE, Golden J. Framing Disease: Studies in Cultural History. New Brunswick, New Jersey: Rutgers University Press, 1992.

12. The National Lung Screening Trial Research

Team, Aberle DR, Adams AM, et al. Reduced lung-cancer mortality with low-dose computed tomographic screening. $N$ Engl J Med 2011;365:395-409.

13. Belcher JR. Thirty years of surgery for carcinoma of the bronchus. Thorax 1983;38:428-32.

14. Berwick DM. The science of improvement. JAMA 2008;299:1182-4

15. Glasziou P, Chalmers I, Rawlins M, et al. When are randomised trials unnecessary? Picking signal from noise. BMJ 2007:334:349-51. 